\title{
Large-Scale Structure in the Universe: Spatial Distribution and Peculiar Ve-
} locities

\author{
Neta A. Bahcall \\ Space Telescope Science Institute \\ 3700 San Martin Drive \\ Baltimore, MD, USA
}

\begin{abstract}
The evidence for the existence of very large scale structures, $\sim 100 h^{-1} \mathrm{Mpc}$ in size, as derived from the spatial distribution of clusters of galaxies is summarized. Detection of $\mathrm{a} \sim 2000 \mathrm{kms}^{-1}$ elongation in the redshift direction in the distribution of the clusters is also described. Possible causes of the effect are peculiar velocities of clusters on scales of $10-100 h^{-1} \mathrm{Mpc}$ and geometrical elongation of superclusters. If the effect is entirely due to the peculiar velocities of clusters, then superclusters have masses of order ${ }^{10}{ }^{16.5} M_{\odot}$ and may contain a larger amount of dark matter than previously anticipated.
\end{abstract}

\section{Introduction}

Rich clusters of galaxies are an efficient tracer of the large scale structure in the universe. Very large structures, of size $\sim 100 h^{-1} M p c$ or more, show up in the distribution of the rich clusters. ( $h \equiv H_{0} / 100 \mathrm{kms}^{-1} \mathrm{Mpc}^{-1}$ is used throughout this paper). Clusters have mean separations of order $50 h^{-1} M p c$ and are therefore efficient in revealing these large scale structures that were not detected previously in the distribution of individual galaxies. The existence of large scale structures became evident by observing strong correlations among clusters, correlations that extend to separations as large as $100 h^{-1}$ Mpc (Bahcall and Soneira 1983; Klypin and Kopylov 1983; Hauser and Peebles 1973), as well as by determining a specific catalog of superclusters in three dimensions (Bahcall and Soneira 1984). Other evidence, such as a giant void ( $\left.300 h^{-1} \mathrm{Mpc}\right)$ of rich clusters (Bahcall and Soneira 1982b), and the extension of some rich superclusters to at least $100 h^{-1} \mathrm{Mpc}$ scale (Bahcall and Soneira 1982a), further support the existence of very large scale structures as a common feature in the universe.

The work was recently extended by Bahcall, Soneira, and Burgett (1986), who used the rich clusters to determine whether peculiar velocities exist on large scales. We find evidence for a large velocity broadening in the redshift distribution that corresponds to a cluster pair velocity of $\sim 2000 \mathrm{kms}^{-1}$. This suggests that dark matter may dominate even on these very large scales. 

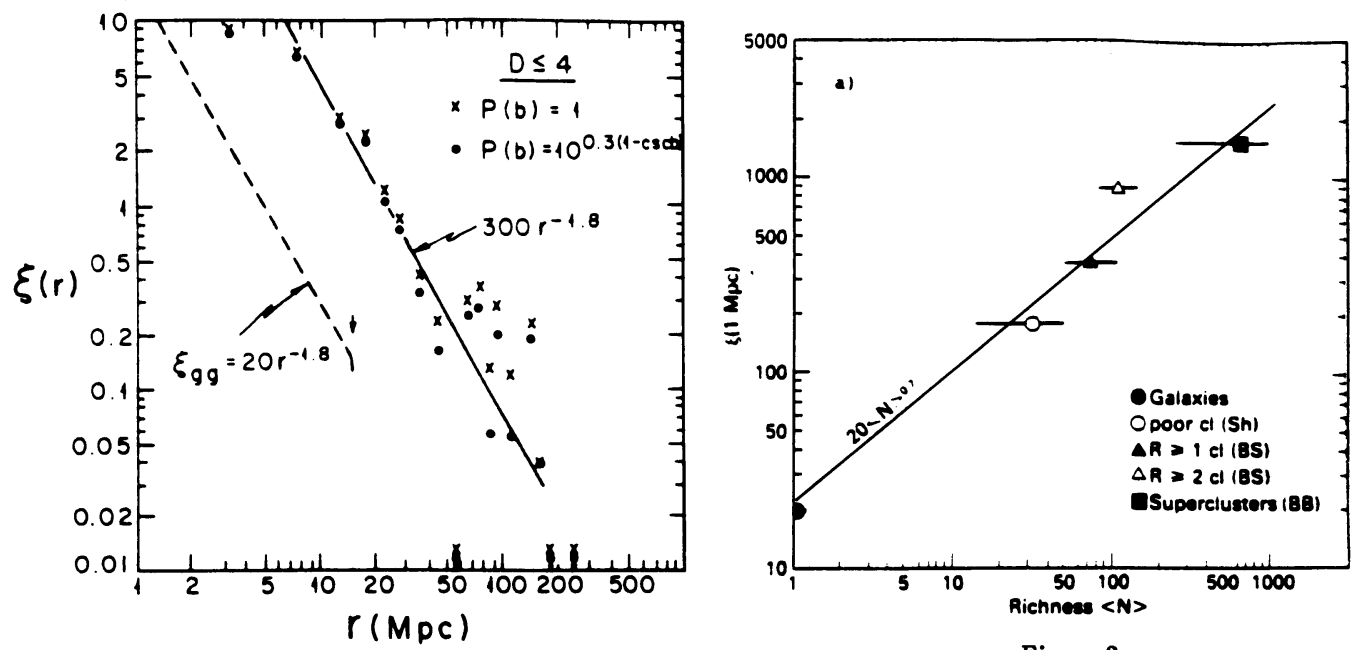

Figure 2

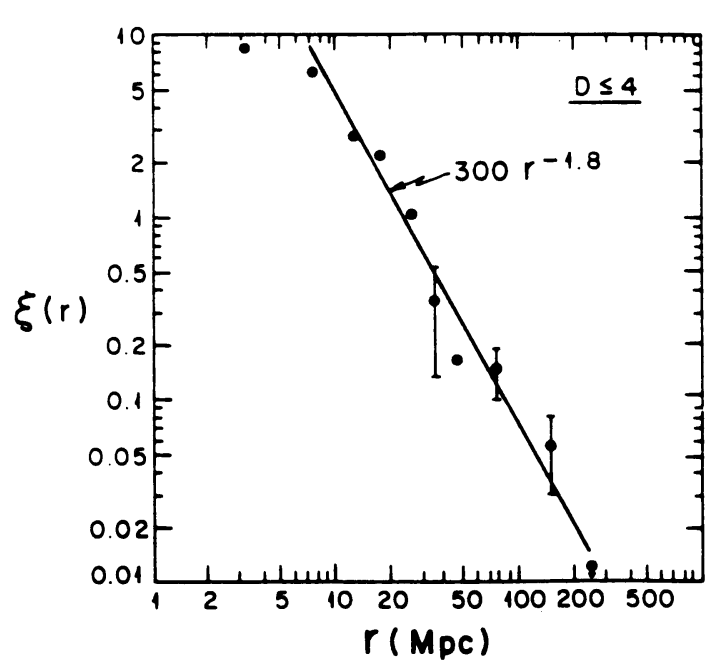

Figure 1

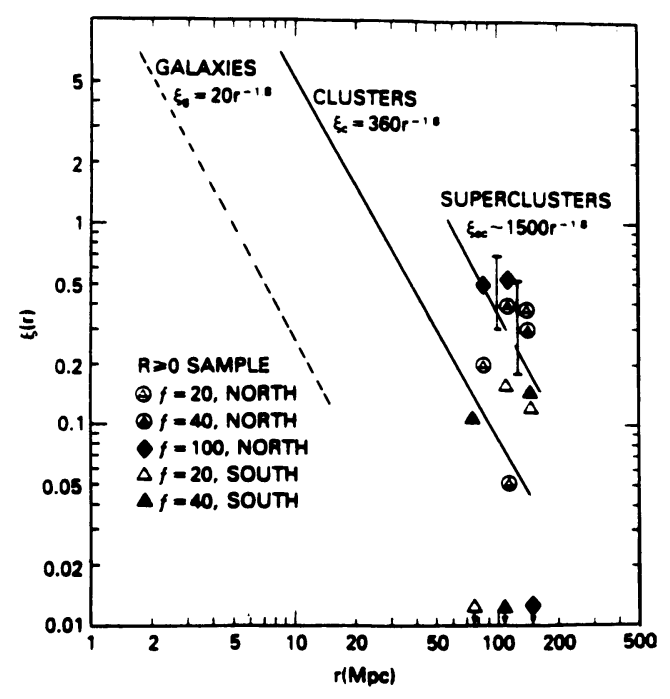

Figure 3

Figure 1: (a) The spatial correlation function of the $D \leq 4$ sample (Bahcall and Soneira 1983). The dashed line is the galaxy-galaxy correlation function determined by Peebles and co-workers. (b) Same as Fig. 1a but plotted in larger bins at large separations.

Figure 2: The dependence of the correlation function on the mean richness ( $\propto$ luminosity) of the system (Bahcall and Burgett 1986). the points are for galaxies, poor clusters (Schectman 1985), $R \geq 1$ and $R \geq 2$ Abell clusters (Bahcall and Soneira 1983), and superclusters (Bahcall and Burgett 1986). The solid line indicates the approximate dependence of richness.

Figure 3: The spatial correlation of superclusters for the $r \geq 0$ sample (Bahcall and Burgett 1986). Different sub-samples are indicated by different symbols. No meaningful correlations are expected below $\sim 50 h^{-1} \mathrm{Mpc}$. 


\section{The Correlation Functions of Clusters and Superclusters}

\section{A. Clusters of Galaxies}

The spatial correlation function of rich clusters of galaxies (Bahcall and Soneira 1983) reveals strong correlations among clusters and implies the existence of structures to scales of at least $\sim 100 h^{-1} \mathrm{Mpc}$. The above investigation is based on studying all rich clusters in the complete redshift sample of the Abell (1958) catalog to distance group

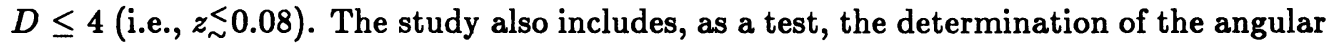
correlation function of all 1651 clusters in the entire Abell catalog to $D \leq 6\left(z_{\sim}^{<0.2)}\right.$.

The cluster correlation function is observed to be approximately 18 times stronger than the correlation function of galaxies (Figure 1). The cluster correlations, $\xi_{c c}$, for Abell richness groups $R \geq 1$ clusters can be expressed as (Bahcall and Soneira 1983):

$$
\xi_{c c}(r)=360 r^{-1.8} \quad r_{\sim}^{<100 h^{-1} M p c}
$$

while the galaxy correlation function (e.g., Groth and Peebles 1977) is represented by:

$$
\xi_{g g}(r)=20 r^{-1.8} \quad r_{\sim}^{<20 h^{-1} M p c}
$$

Bahcall and Soneira (1983) also found that the correlation function depends strongly on cluster richness, with rich clusters $(R \geq 2)$ showing stronger correlations by a factor of $\sim 3$ as compared with the poorer $(R=1)$ clusters (both are consistent with an $r^{-1.8}$ power law). This result, combined with the lower correlation amplitude of individual galaxies lead the authors to the conclusion that progressively stronger correlations exist, at a given separation, for richer galaxy systems. This trend of increasing correlation strength with richness is shown in Figure 2. A recent study by Shectman (1985) of the correlations of still poorer clusters appears to be consistent with the trend suggested by Bahcall and Soneira. A phenomenological model that can explain the observed increase of correlation strength with richness was recently proposed by Bahcall (1986) and is summarized in section IV.

A representation of the observed correlations as a universal dimensionless correlation function, and its implication for a scale invariant clustering process is discussed in section III.

\section{B. Superclusters}

Bahcall and Burgett (1986) carried the study of rich galaxy clusters one step further by studying the spatial distribution of superclusters. The sample used was the Bahcall-Soneira (1984) complete catalog of superclusters to $z \leq 0.08$, where superclusters are defined as groups of rich clusters and identified by a spatial density enhancement of clusters. All volumes of space with a spatial density of clusters $f$ times larger than the mean cluster density are identified in the above catalog as superclusters for a specified value of $f$. The supercluster selection process was repeated for various overdensity values $f$, from $f=10$ to $f=400$, yielding specific supercluster catalogs for each $f$ value. A 
total of 16 superclusters are cataloged for $R \geq 1$ and $f=20$, and 26 superclusters for $R \geq 0$ and $f=20$. The spatial correlation among the superclusters was determined for the samples of different richness and overdensity values. Because of the large size of the superclusters themselves, no meaningful correlations are expected at small separations $\left(<50 h^{-1} \mathrm{Mpc}\right)$. In addition, no detectable correlations are expected at very large separations ( $>200 h^{-1} \mathrm{Mpc}$ ) since this scale is comparable to the limits of the sample. Any observable correlations are therefore expected only in a separation "window" around $\sim 100 h^{-1} \mathrm{Mpc}$.

The results, presented in Figure 3, reveal correlations among superclusters on a very large scale: $\sim 100-150 h^{-1} M p c$. Because of the small size of the supercluster sample, the statistical uncertainty is appreciable; the observed effect is at the $3 \sigma$ level (as compared with numerical simulations of random catalogs). In addition, all the samples with different overdensities and cluster richnesses show a similar effect at a similar scale length. The results imply the existence of very large scale structures with scales of $\sim 100-150 h^{-1} \mathrm{Mpc}$.

Figure 3 also shows that the supercluster correlation strength is stronger than that of the rich cluster correlations by a factor of approximately 4. It is approximately two orders of magnitude stronger than the galaxy correlation amplitude. While this enhancement is observed in the $\sim 100-150 h^{-1} \mathrm{Mpc}$ range, it is possible that the supercluster correlation function also follows an $r^{-1.8}$ law. If the correlations follow an $r^{-1.8}$ law, then the function would satisfy the relation

$$
\xi_{s c, s c}(r) \simeq 1500 r^{-1.8} \simeq(r / 60)^{-1.8} .
$$

The implied correlation scale of superclusters would be $60 h^{-1} \mathrm{Mpc}$, as compared with $5 h^{-1} M p c$ for the correlation scale of galaxies (Groth and Peebles 1977) and $25 h^{-1} M p c$ for rich $(R \geq 1)$ clusters (Bahcall and Soneira 1983). This increase is correlation strength is consistent with the earlier prediction of Bahcall and Soneira (1983) of increased correlations with richness (luminosity) of the system. The supercluster correlation amplitude fits well the predicted correlation curve (Figure 2).

\section{A Universal Correlation Function}

The increase of correlation strength with richness (Figure 2) implies that rich, luminous systems are more strongly clustered, at a given separation, than poorer systems. The power-law of the correlation functions is also observed to be identical in the various systems studied. Either initial conditions, or superceding evolution, may be responsible for these observed clustering phenomena.

In Figure 4 we plot the amplitude of the correlation functions of the various systems (galaxies, poor and rich clusters, superclusters) as a function of the mean separation of objects in the sample, $d$ (see Bahcall and Burgett 1986). The mean separation is related to the mean spatial density of objects in the sample, $n$, through $d=n^{-\frac{1}{3}}$. It is apparent from Figure 4 that the correlation strength increases with the sample's mean separation. Moreover, a dimensionless correlation function normalized to the sample's mean separation, $d$, appears to yield a constant, universal function for all systems studied 


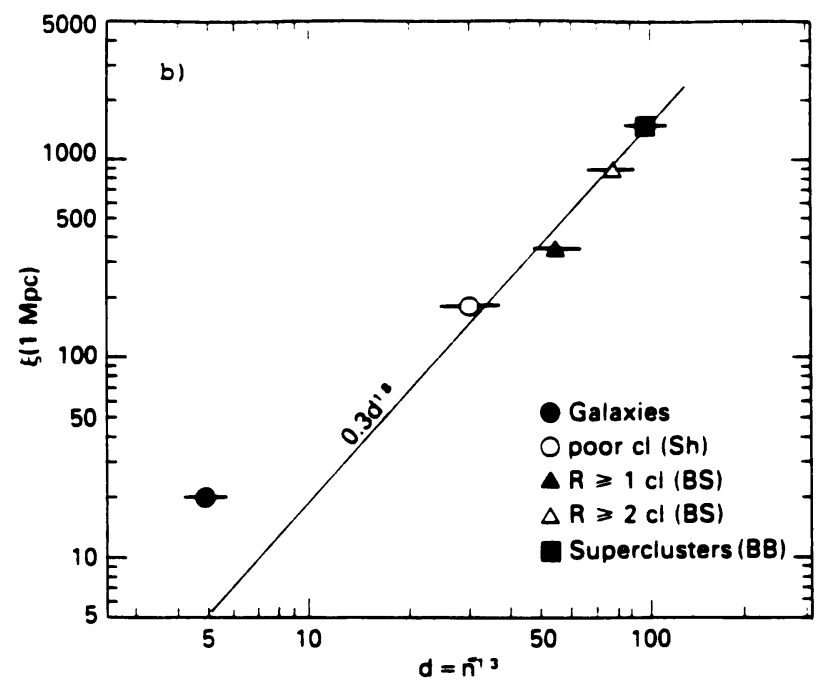

$\underline{\text { Figure } 4}$

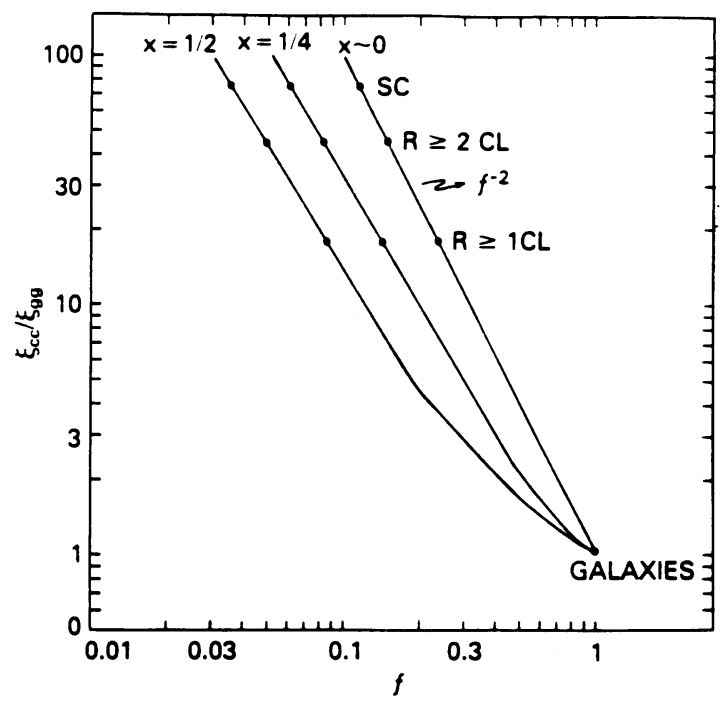

(FRACTION OF GALAXIES IN CLUSTERS)

Figure 5

Figure 4: The dependence of the correlation function on the mean separation of objects in the system (Bahcall and Burgett 1986). The indicated ranges in the mean separation represent a $\pm 50 \%$ variation in the mean density $n$ (where $d=n^{-\frac{1}{3}}$ ). The solid line represents a $d^{1.8}$ dependence.

Figure 5: The ratio of the cluster to galaxy correlation functions predicted from Bahcall's (1986) model (eq. 5) is plotted as a function of the fraction $f$ of galaxies associated with the clusters. The different curves represent different values of the "field" (non-cluster) correlation strength, $\xi_{g g}^{f}$. In terms of the ratio parameter $x=\xi_{g g}^{f} / \xi_{g g}$, the curves represent $x=0, \frac{1}{4}$, and $\frac{1}{2}$, as indicated. The observed correlation strengths of $R \geq 1$ clusters, $R \geq 2$ clusters, and superclusters is indicated by the points. 
(with some enhancement for galaxies, as described below). This universal dimensionless correlation function has the form

$$
\xi_{i}(r) \simeq 0.3\left(r / d_{i}\right)^{-1.8},
$$

where the index $i$ refers to the system being considered, and $d_{i}$ is its mean separation. The correlation function of galaxies is stronger than given by equation (4) by a factor of about four (see also Figure 4).

The universality of the correlation function implies a scale-invariant clustering process (Szalay and Schramm 1985). The stronger dimensionless galaxy correlations may imply gravitational enhancement on smaller scales. If a non-linear process, other than gravity, participates in galaxy formation, and this process is scale-invariant, the created structure will have a single power-law correlation function, the slope of which is related to the geometry of the structure, i.e., its fractal dimension. Small scale gravitational clustering may break the scale invariance and increase the dimensionless correlation amplitude for galaxies.

Cosmic strings, serving as the primary agent in the formation of galaxies and clusters was recently suggested as one model for such a scale-invariant infrastructure (Turok 1986). The model yields a scale-invariant correlation function similar to that observed, with a power-law of $\mathbf{- 2}$.

\section{A Phenomenological Clustering Model}

A phenomenological model that explains the observed difference between the galaxy and rich cluster correlation functions was recently proposed by Bahcall (1986). The model is based on the fraction of galaxies that participate in the clustering, and explains the observed trend of increased correlation strength with richness. A summary of the model is presented below.

The galaxy correlations depend, at least partially, on the rich cluster correlations since clusters contain galaxies. It can be shown that if all galaxies were members of rich clusters, the two correlation functions should be approximately the same on large scales. In this case they both trace the same large scale structure. The fraction of galaxies in clusters is clearly less than unity. The effect of the fraction of galaxies, $f$, that are associated with rich clusters, on the relation between the cluster and galaxy correlation functions, is the basis for the model. The parameter $f$ represents the probability that a randomly chosen galaxy is correlated with a rich cluster. These associations may include large structures (separations of tens of $M p c$ ), comparable to the separations observed in the cluster correlation function (and well above the standard Abell radius of $1.5 h^{-1} M p c$ ).

The galaxy correlation function contains contributions from three terms: galaxy pairs from the fraction $f$ of galaxies that are cluster members; pairs from the fraction 1- $f$ of galaxies that are non-cluster members ("field"); and cross-term pairs. Inserting the analytic expressions for each of these terms into the expression for the overall galaxy correlation function yields: 


$$
\left(\frac{\xi_{c c}}{\xi_{g g}}\right)^{\frac{1}{2}}=\frac{1-(1-f)\left(\xi_{g g}^{f} / \xi_{g g}\right)^{\frac{1}{2}}}{f}
$$

The above ratio of the cluster to galaxy correlation strength depends on two parameters: the fraction of galaxies in clusters, $f$, and the ratio of the "field" galaxy correlation strength, $\xi_{g g}^{f}$ (i.e., the correlation of the 1- $f$ fraction of galaxies outside the rich clusters) to the overall galaxy correlation $\xi_{g g}$. If all galaxies were associated with rich clusters, i.e., $f=1$, then the galaxy and cluster correlations are identical, as expected. However, for any fraction $f<1$, the galaxy correlations will be smaller than the parent cluster correlations due to the reducing effect of the less clustered "field" galaxies. Figure 5 represents graphically relation (5). The curves are the expected $\xi_{c c} / \xi_{g g}(f)$ relations for selected values of the parameter $x \equiv \xi_{g g}^{f} / \xi_{g g}$.

The observed correlation strengths discussed in sections II and III are represented by the data points. The observed ratio $\xi_{c c} / \xi_{g g} \simeq 18$ for $R \geq 1$ clusters yields a fraction of galaxies in clusters that ranges from $f \simeq 25 \%$ for $\xi_{g g}^{f} / \xi_{g g} \simeq 0$ to $f \simeq 15 \%$ for $\xi_{g g}^{f} / \xi_{g g} \simeq \frac{1}{4}$. Therefore, if approximately $20 \%$ of all galaxies are associated with rich $(R \geq 1)$ clusters, the galaxy correlation function will be, as observed, $\sim 18$ times weaker than the cluster correlations.

The model suggests that the fraction of galaxies associated with rich clusters is considerably larger than previously expected; most of these galaxies are distributed in the outer tails of the clusters, which may extend to at least $\sim 30 h^{-1} M p c$. Most clusters are therefore predicted to be embedded within much larger structures.

The model makes testable predictions that can be studied with complete redshift surveys. The model may also explain the negligible correlations observed among $L y-\alpha$ clouds in QSO spectra (Sargent et.al. 1980). If these clouds cannot exist in rich clusters due to the high pressure in the latter, and therefore belong mostly to the non-cluster component, negligible correlations would be expected for the clouds.

\section{Peculiar Velocities on Large Scales}

The discussion in the previous sections summarizes evidence for the general existence of structures on the scale of $\sim 10-150 h^{-1} M p c$. A question of critical importance is what are the velocity fields in these structures. Peculiar velocities of clusters on these scales may indicate the existence of large amounts of (dark) matter. Recently, Bahcall et.al. (1986) used the complete redshift sample of rich clusters (§II) to study the possible existence of peculiar motion and/or structural anistropy on large scales. They find strong broadening in the redshift distribution that corresponds to a cluster pair velocity of $\sim 2000 \mathrm{kms}^{-1}$. These findings are summarized below.

The distribution of clusters in space was studied by separating the three-dimensional distribution into its components along the line-of-sight (redshift) axis and the perpendicular axes projected on the sky. All clusters were assumed to be located at their Hubble distances as indicated by their redshifts. A cartesian coordinate system was set up in which cluster pair separations in $M p c$ were determined. The $z$-axis of the system was defined along the line-of-sight direction, and the $x$ and $y$ axes were in the plane projected 

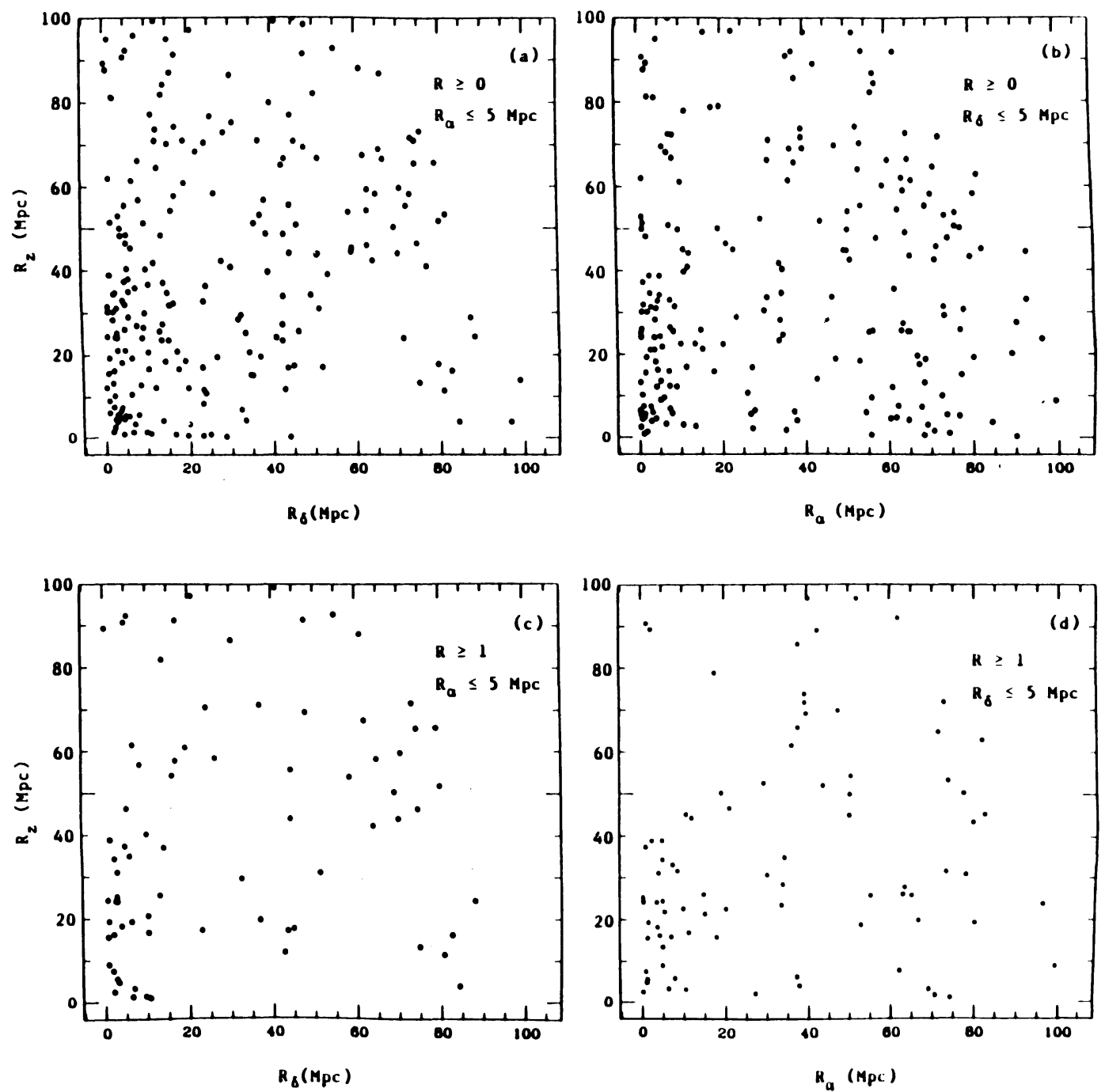

Figure 6: Scatter-diagrams of cluster pair separations in $M p c$ in the $R_{z}-R \alpha$ and $R_{z}-R \delta$ planes (Bahcall et.al. 1986). Figure 6a-6b and $6 \mathrm{c}-6 \mathrm{~d}$ represent, respectively, the $R \geq 0$ and $R \geq 1$ richness samples. The elongation in the redshift direction is apparent in all cases. 
on the sky. For simplicity, we used the $\alpha$ and $\delta$ coordinate system as the $x$ and $y$ axes, respectively. A scatter-diagram of the cluster pair separations in the $z$ direction $\left(R_{z}\right)$ versus their separations in $\alpha$ or $\delta\left(R_{\alpha}\right.$ or $\left.R_{\delta}\right)$ was then determined for each sample. For a meaningful comparison between any two components, the third component was restricted to a given small value, which was varied in order to check the dependence of the results on the choice of the limit of the third component. In addition, only pairs with a total spatial separation less than $100 h^{-1} M p c$ (i.e. $R_{\alpha}^{2}+R_{\delta}^{2}+R_{z}^{2} \leq 100 h^{-1} M p c$ ) were considered. This restriction limits the outer bounds of the sample studied but has no effect on the relevant inner points.

If all clusters were located at their Hubble distances with negligible peculiar motion, and if the sample was not dominated by elongated structures in a given direction, a symmetric scatter-diagram should be observed. In this case, a similar distribution should be observed along each of the three axes. If a large peculiar velocity exists among clusters, it would manifest itself as an elongated distribution along the $z$-direction in the $R_{z}-R_{\alpha}$ and $R_{z}-R_{\delta}$ diagrams. Such an elongation is normally interpreted as peculiar motion. However, the effect may also be caused by geometrically elongated structures, if they dominate the sample (with elongation toward the $z$-direction; see below).

Our results are presented in Figures 6 to 8 . The scatter-diagrams are plotted in Figure 6 in both the $R_{z}-R_{\alpha}$ and $R_{z}-R_{\delta}$ planes, for both the $R \geq 0$ and $R \geq 1$ samples. Frequency distribution histograms representing these diagrams are presented in Figure 8. A strong and systematic elongation in the $z$-direction exists in all the real samples studied. Scatter-diagrams for sets of random catalogs were also generated for comparison; two representative examples are shown in Figures 7a-7b. As expected, no conspicuous elongation appears in any of the random catalogs. As an additional test, we have also determined the scatter diagrams in the projected plane, $R_{\alpha}-R_{\delta}$, of the cluster samples; typical results are shown in Figures 7c-7d. Again, as expected, a symmetric distribution is observed in this plane. These tests strengthen the conclusion that the observed elongation is real. The effect of elongation is strong; statistically it corresponds to approximately $8 \sigma$ in a single sample (assuming, for illustrative simplicity, Gaussian statistics). It is therefore unlikely that the observed redshift elongation is a chance fluctuation. The effect becomes more apparent in the larger $R \geq 0$ sample; this is expected if the effect is real.

To determine what velocity could cause the observed effect, we convolved the frequency distribution observed along the projected axis, which is unperturbed by peculiar motion, with a Gaussian velocity distribution. This convolved distribution should match the broadened distribution observed in the redshift direction. The best fit is obtained for a Gaussian velocity width of $\sqrt{2} \sigma \simeq 2000 \mathrm{kms}^{-1}$. The fits are shown as the smooth curves in Figure 8.

What causes the elongation? One (standard) interpretation is peculiar motion. Another possibility is a true geometrical elongation in the shape of the large-scale structures. I discuss below both possibilities.

\section{A. Peculiar Velocity Among Clusters}

To estimate a supercluster mass which may support this velocity, we use a typical 

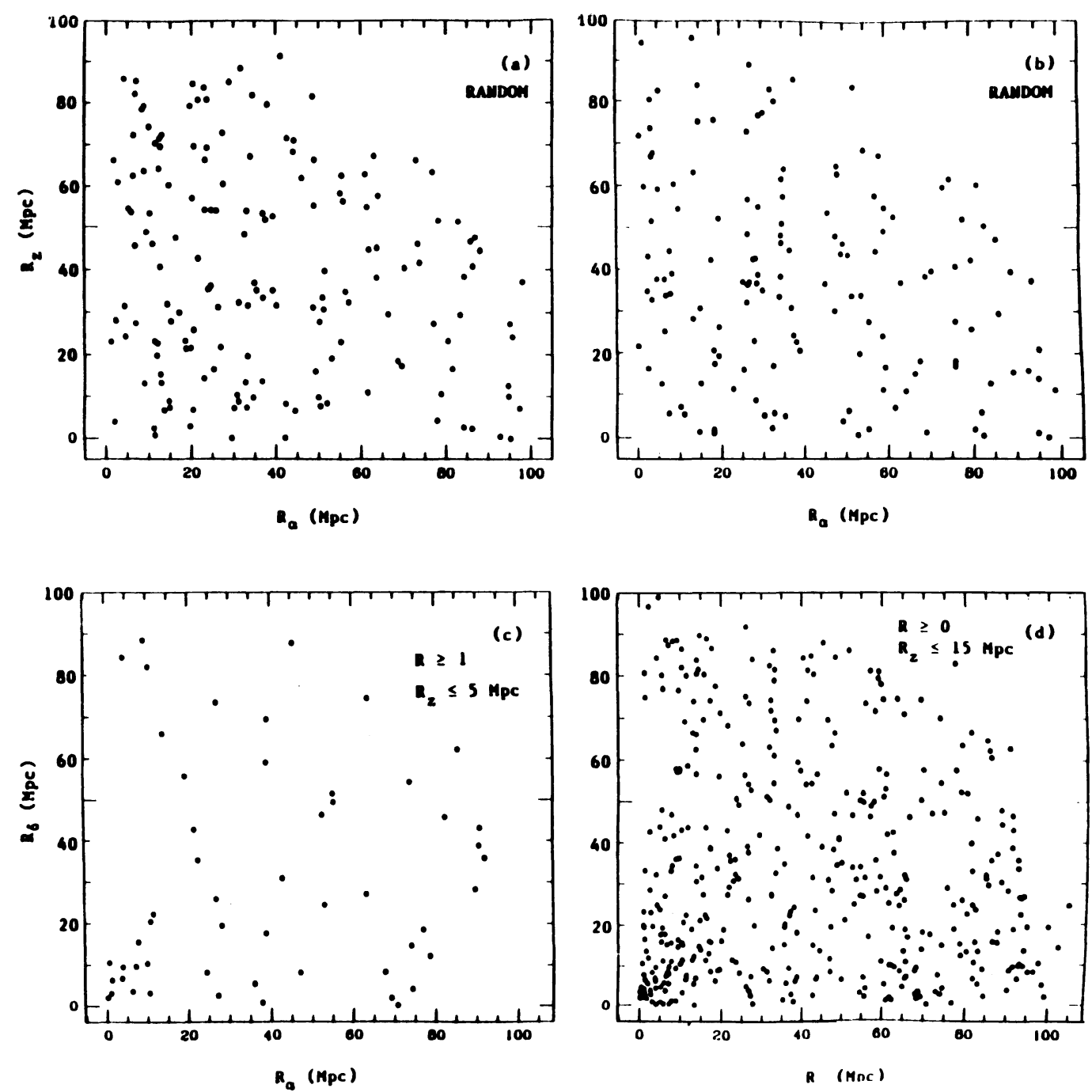

Figure 7: Same as Figure 6 but for typical random distribution of clusters (Fig. 7a-7b), and for the projected distribution (i.e. $R_{\delta}-R_{\alpha}$ plane) of the actual cluster samples (Fig. 7c-7d). No elongation is expected in either case and none is observed. The clustering of clusters is apparent in the data sample of Fig. $7 \mathrm{c}-7 \mathrm{~d}$. 


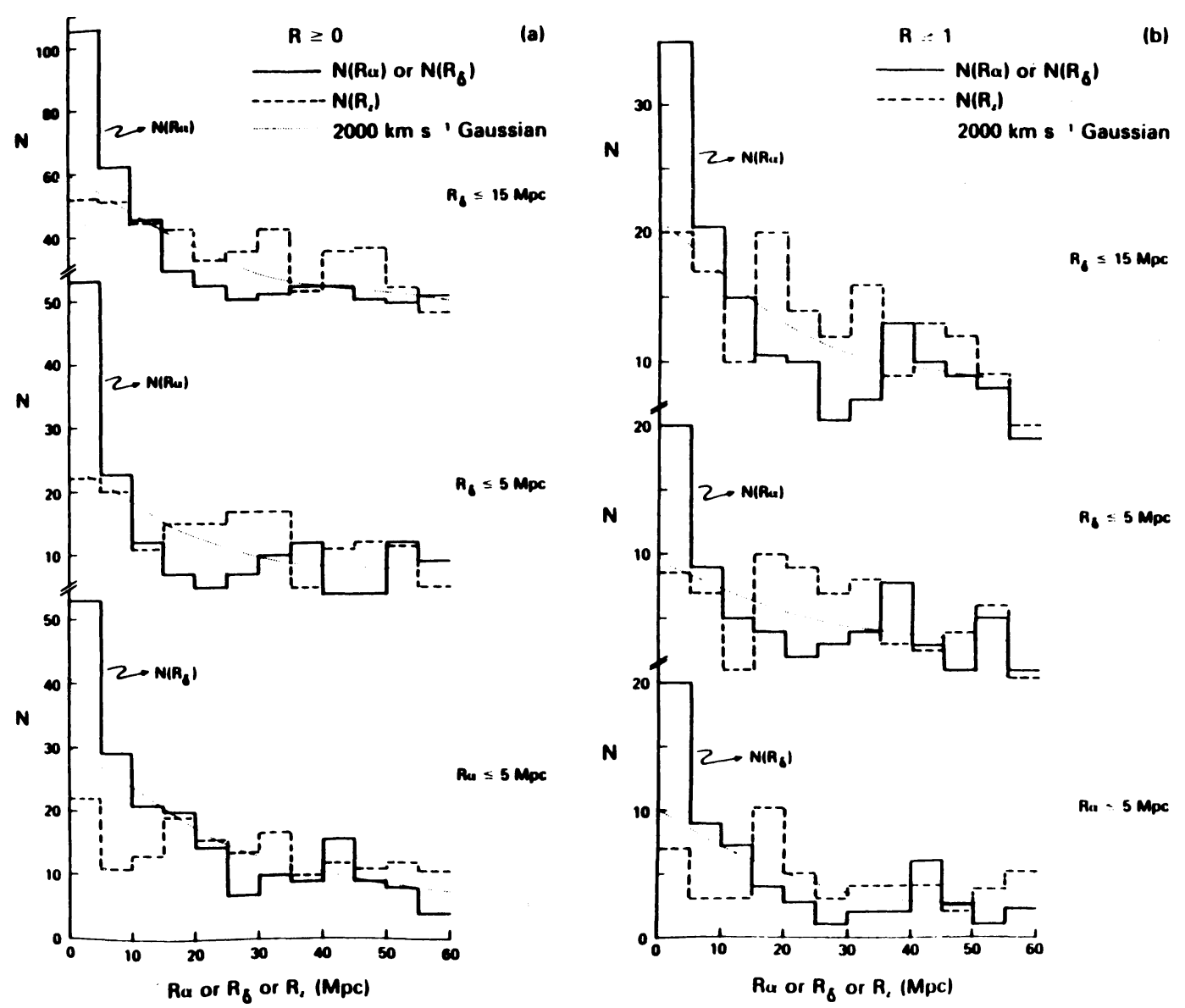

Figure 8: Histograms representing the distribution of pairs along the redshift, $R_{x}$, and projected, $R_{\alpha}$ and $R_{\delta}$, directions, as determined from the scatter-diagrams, are shown for the $R \geq 0$ (Fig. 8a) and $R \geq 1$ (Fig. 8b) samples. The number of cluster pairs as a function of projected separation, $N\left(R_{\alpha}\right)$ or $N\left(R_{\delta}\right)$, is represented by the solid histogram. The number of clustered pairs in the redshift direction, $N\left(R_{z}\right)$, is represented by a dashed histogram. The dotted curve represents a convolution of the projected distribution $\left(N\left(R_{\alpha}\right)\right.$ or $\left.N\left(R_{\delta}\right)\right)$ with a Gaussian of $2000 \mathrm{kms}^{-1}$ width. 
supercluster size of $\sim 25 h^{-1} M p c$ (=cluster correlation scale-length) and assume the virial relation $M \propto v^{2} r$. This yields a typical supercluster mass of

$$
M_{s c} \simeq 3 \times 10^{16} M_{\odot}
$$

This mass is comparable to the mass of $\sim 30$ rich clusters while typically only $\sim$ 3-5 rich clusters are members of a supercluster. Even when the luminous tails of clusters are accounted for the result may still imply an excess of dark matter in superclusters as compared with clusters. Using an observed luminosity and/or density profile of $r^{-3}$ or $r^{-2.5}$ around a rich cluster, we estimate an $\mathrm{M} / \mathrm{L}$ for superclusters that is typically two to three times that of rich clusters, i.e., $M / L \sim 500$.

\section{B. Geometrical Elongation of Superclusters}

The elongation observed in the scatter diagrams may also be caused, at least partially, by a geometrical elongation of superclusters. If the most prominent superclusters are elongated toward the line-of-sight direction, an apparent elongation in the distribution of pair separation along this axis may result. This preferred direction of elongation may occur either by chance, in a small sample of superclusters, or in cases of specific structures such as large cells or shells, when the most distant face-on components lie beyond the redshift limit of the sample. In this latter case, the sample may be dominated by large cells $\left(\sim 100 h^{-1} M p c\right)$ that are cut off by the sample's redshift limit near their outermost edge. Accurate distance indicators such as Tully-Fisher type relations are needed in order to distinguish between peculiar velocity and geometrical elongation of large scale structures.

\section{$\underline{\text { References }}$}

Abell, G.O., 1958, Ap.J.Suppl. 3, 211

Bahcall, N.A., 1986, Ap.J.Letters, 302, L41.

Bahcall, N.A., and Burgett, W., 1986, Ap.J.Letters 300, L35.

Bahcall, N.A., and Soneira, R.M., 1982a, Ap.J.Letters 258, L17.

Bahcall, N.A., and Soneira, R.M., 1982b, Ap.J. 262, 419.

Bahcall, N.A., and Soneira, R.M., 1983, Ap.J. 270, 20.

Bahcall, N.A., and Soneira, R.M., 1984, Ap.J. $277,27$.

Bahcall, N.A., and Soneira, R.M., and Burgett, W.S., 1986, Ap.J. 311, (December 1).

Groth, E., and Peebles, J.P.E., 1977, Ap.J. 217, 385.

Hauser, M.G., and Peebles, P.J.E., 1973, Ap.J. 185, 757.

Klypin, A.A., and Kopylov, A.I., 1983, Soviet Astronomy Letters, 9, 41.

Sargent, W.L.W., Young, P.J., Boksenberg, A., Tytler, D., 1980, Ap.J.Suppl. 42, 41.

Schectman, S., 1985, Ap.J.Suppl. 57, 77.

Szalay, A.S., and Schramm, D.N., 1985, Nature 314, 718.

Turok, N. 1986, preprint. 


\section{DISCUSSION}

BURNS: (1) $R=0$ clusters can be very useful in tracing supercluster structure if selected carefully (in terms of distance) and restricted to certain uses. In particular $\mathrm{R}=0$ clusters connect $r$ ich superclusters together as in Hercules. (2) Can these $\sim 2000 \mathrm{~km} / \mathrm{sec}$ peculiar velocities between clusters effectively "wash out" the claimed supercluster and void structures in the distribution of Abell clusters?

BAHCALL: (1) One should be very careful when using any sample which is incomplete, such as $\mathrm{R}=0$ clusters. Any project that requires completeness, or that may be sensitive to incompleteness effects, such as correlation functions, percolations, or the statistical determination of structure and shape, could be highly affected by such incompleteness, and meaningful interpretation may be difficult. They may be used only as an additional check, or trend, of the complete sample results.

(2) The $2000 \mathrm{~km} \mathrm{~s}^{-1}$ between pairs of clusters in superclusters may be due to either velocity or actual geometrical elongation, as I described. If a large part of the effect is indeed due to motions, it would obviously distort any interpreted geometry and shape of the large structures that do not account for such velocities. The reverse, of course, is also true, i.e., geometry will distort velocities.

KARACHENTSEV: I think we must be very careful with the "universal" slope of correlation functions. For the centers of $\sim 500$ binary galaxies a correlation function has the power law parameter -1.0 , but not -1.8 , as for standard ones by Peebles. This difference is not caused only by selection effects.

BAHCAIL: Yes. The "universal" correlation function applies only on large scales, from poor clusters, to richer clusters, superclusters etc. It does not apply on the small scale of galaxies and binaries, where gravitational forces are expected to yield additional clustering. Indeed, as I briefly noted, the galaxy correlation function is approximately three times stronger than the universal function on the large-scale.

DENG: How many clusters are in your sample?

BAHCALL: The complete statistical redshift survey used for the direct spatial correlation function determination contains 104 clusters to $z \leqslant 0.1$. However, it is tested and compared with the full sample of almost 2000 clusters to $z \leqslant 0.2$, using the angular correlation 
function, where the scaling scales exactly as expected. Therefore, the effective number is probably close to 2000 clusters. For the velocity dispersion determination, we used both the 104 clusters in the complete $R \geq 1$ redshift sample, as well as the larger $R \geq 0$ sample of $\sim 200$ clusters with measured redshifts. 